\title{
Stator Winding Short Circuit Fault Detection based on Uncertainty Ellipsoid Intersection for Three Phase Induction Motors
}

\author{
Mohammed Obaid Mustafa and George Nikolakopoulos \\ Control Engineering Group, Division of Systems and interaction, Department of Computer Science, \\ Electrical and Space Engineering, Luleå University of Technology, SE- 97187 Luleå, Sweden \\ \{mohoba,geonik\}@ltu.se
}

Keywords: Three Phase Induction Motor, Fault Detection and Diagnosis, Set Membership Identification, Uncertainty Ellipsoid Intersection, Geometrical Analysis.

Abstract: In this article a fault detection scheme for different percentage of stator winding short circuit in one phase of three phase induction motors is presented. In the examined case, the induction motor in the faulty and healthy case has been transformed in the two phase $(q-d)$ model. The model has been identified by the utilization of a Least Squares Set Membership Identification (SMI) algorithm, where additional to the identified parameters, confidence intervals can be also calculated, based on a priori knowledge for the corrupting measurement noise. The identified confidence intervals in an $\mu$-dimensional space can be represented as hyper-ellipsoids having as a center the identified parameters' vector. The novelty of this article stems from the proposal of a fast and geometrical based scheme, which relies on the calculation of the distance among centers of hyperellipsoids and the corresponding intersection in each iteration of the identification procedure. Detailed analysis of the proposed fault detection strategy, as also extended simulation results are being presented that prove the efficiency of the suggested scheme.

\section{NOMENCLATURE}

$V_{q s}, V_{d s}:$ Stator voltages Quadrature frame $(V)$

$i_{q s}, i_{d s}:$ Stator currents Quadrature frame $(A)$

$r_{s}, r_{r}$ : Resistance of stator's and rotor's winding $(\mathrm{Ohm})$

$L_{S}, L_{r}:$ Stator's and rotor's self inductance (Henry)

$L_{m}$ : Mutual inductance (Henry)

$\omega_{r}:$ Rotor's angular speed $(\mathrm{rad} / \mathrm{sec})$

$\omega_{m}:$ Rotor's speed (mechanical) $(\mathrm{rad} / \mathrm{sec})$

$\omega_{s}:$ Supply angular frequency $(\mathrm{rad} / \mathrm{sec})$

$P$ : No. of poles pairs

$J$ : Moment of inertia $\left(\mathrm{Kg} \cdot \mathrm{m}^{2}\right)$

$T_{L}:$ Load torque $(\mathrm{Nm})$

$T_{e}$ : Electromagnetic torque $(\mathrm{Nm})$

$q:$ Quadrature axis frame

$d:$ Direct axis frame

$s:$ Stator quantities

$r:$ Stator quantities

\section{INTRODUCTION}

Three phase induction motors are very important in different applications in industrial and power systems (F. Jawad, 2009) as it is commonly known that induction motors have good properties such as highly reliability, require low maintenance, and have high efficiency. Therefore, the condition monitoring of these electrical machines has received considerable attention in recent years as fault detection and diagnosis are very important to reduce the maintenance cast and prevent downtimes for electrical drive systems (B. Mirafzal, 2006).

Induction motors have many type of faults, while some of these fault happen in the stator or in the rotor, such as short circuit in stator winding, broken rotor bar, and bearing faults (Nandi and Toliyat, 2005). Stator winding consists of coils of insulated copper wire placed in the stator slots, while the stator winding faults start due to the insulation breakdown between two adjacent turns in a coil for the same phase; this fault is usually called a turn-to-turn fault or interturn short circuit. Therefore, this type of fault is very serious as it will produce an extra heat, and it will create an imbalance in the magnetic filed of the machine. In fact, the majority of these defects are due to a combination of various stresses acting on the stator, which can be classified into thermal, electrical, mechanical, and environmental factors.

Until now, various scientific methods have been proposed in the field of fault detection and diagno- 
sis for induction motors such as: a) Artificial Neural Networks (Gaeid and Mohamed, 2010), b) Fast Fourier Transform (Bachi et al., 2006), c) Time Step Coupled Finite Element-State Space (Gaeid and Mohamed, 2010), d) Motor Current Signature Analysis (Aydin et al., 2011), e) Wavelets, and Complex Park Vectors (Gaeid and Mohamed, 2010).

All of these methods base their operation on spectral analysis of stator currents, stator voltages, and electromagnetic torque (Aydin et al., 2011), while are able to provide a faster detection and identification of the fault, but will lead to incorrect conclusions in some cases, such as low load conditions, in transient situations or in perturbed environments (i.e. fluctuating load torque and unideal supply). From another point of view Independent Component Analysis (ICA) and Support Vector Machines (SVMs) have been applied to detect and diagnose of induction motor faults (Widodo et al., 2007; A. Widodo, 2008) as alternative approaches to the previous mentioned cases.

The novelty and the main scientific contribution of this article stems from: a) the combination of the SM-identification technique for calculating recursively the motor parameters and the corresponding uncertainty bounds based on the assumed noise levels, with b) the proposal of a fault detection scheme based on fundamental geometrical properties of the calculated parameter bounding uncertainty. More analytically, in this article the problem of fault detection is transformed to the geometrical problem of representing uncertainty into $\mu$-dimensional hyper-ellipsoid spaces and examining basic properties of the resulting $\mu$-dimensional spaces, such as center of ellipsoid, ellipsoidal intersection, and distance from the centers. The proposed scheme has a low complexity and as it is going to be presented in the sequel, it can be directly transferred to real life implementations.

The rest of the article is being structured as it follows. In Section 2 the model derivation and simplification, for the healthy and the faulty cases is being derived. In Section 3 the SMI scheme is being presented, followed by the proposed fault conditioning framework in Section 4. Section 5 contains multiple simulation results that prove the efficacy of the proposed methodology, while the conclusions are drawn in the last Section 6.

\section{INDUCTION MOTOR MODELING}

\subsection{Healthy Case}

The state space form for the three phase induction motor can be represented as it follows: (Vas, 1992; Mustafa et al., 2012b)

$$
\left[\begin{array}{c}
\frac{d i_{q s}}{d t} \\
\frac{d i_{d s}}{d t} \\
\frac{d i_{q r}}{d t} \\
\frac{d i_{d r}}{d t}
\end{array}\right]=A\left[\begin{array}{c}
i_{q s} \\
i_{d s} \\
i_{q r} \\
i_{d r}
\end{array}\right]+B\left[\begin{array}{c}
V_{q s} \\
V_{d s} \\
0 \\
0
\end{array}\right]
$$

where:

$$
\begin{aligned}
\mathbf{A} & =\frac{1}{\delta}\left[\begin{array}{cccc}
-L_{r} r_{s} & 0 & L_{m} r_{s} & 0 \\
0 & -L_{r} r_{s} & 0 & L_{m} r_{s} \\
L_{m} r_{r} & 0 & -L_{s} r_{r} & w_{r} \delta \\
0 & L_{m} r_{r} & -w_{r} \delta & -L_{s} r_{r}
\end{array}\right] \\
\mathbf{B} & =\frac{1}{\delta}\left[\begin{array}{cccc}
L_{r} & 0 & -L_{m} & 0 \\
0 & L_{r} & 0 & -L_{m} \\
-L_{m} & 0 & \mathrm{E}_{s} & 0 \\
0 & -L_{m} & 0 & L_{s}
\end{array}\right]
\end{aligned}
$$

with $\delta$ defined as:

$$
\delta=L_{s} L_{r}-L m^{2}
$$

while the motor's torque and angular speed, in case that the fraction is being neglected, is denoted as:

$$
\begin{aligned}
T_{e} & =\frac{3}{2} P L_{m}\left[i_{q s} i_{d r}-i_{q r} i_{d s}\right] \\
J \frac{d \omega_{m}}{d t} & =T_{e}-T_{L}
\end{aligned}
$$

\subsection{Stator Winding Short Circuit Modeling}

The focus of this research effort is on the stator faults during short circuit between stator winding, which happen in one phase of the motor. In the examined case all the stator parameters are considered to be identical when short circuit happens in the winding of the three phase induction motor, while both stator's resistance and inductance, as also the mutual inductances between stator and rotor will be directly affected. In the case of such a fault, the modified (faulty) versions of the matrices $\mathbf{A}$ and $\mathbf{B}$ in equation (1), will be changed to $\mathbf{A}^{*}$ and $\mathbf{B}^{*}$ and become as:

$$
\begin{aligned}
& \mathbf{A}^{*}=-\mathbf{R}_{\mathbf{f}}^{*} \mathbf{L}_{\mathbf{f}}^{*-1} \\
& \mathbf{B}^{*}=\mathbf{L}_{\mathbf{f}}^{*-1}
\end{aligned}
$$


while the inductance matrix in the faulty case will become as (Chen and Zivanovic, 2009):

$$
\mathbf{L}_{\mathbf{f}}^{*}=\left[\begin{array}{cccc}
L_{11} & 0 & L_{14} & 0 \\
0 & L_{22} & 0 & L_{25} \\
L_{41} & 0 & L_{44} & 0 \\
0 & L_{52} & 0 & L_{55}
\end{array}\right]
$$

The elements of $\mathbf{L}_{\mathbf{f}}^{*}$ are being defined as (Chen and Zivanovic, 2009):

$$
\begin{aligned}
& L_{11}=\frac{1}{3}\left(g_{a s}+1\right) L_{s}+\frac{1}{9}\left(2 g_{a s}+1\right)^{2} L_{s} \\
& L_{14}=L_{41}=\frac{1}{3}\left(g_{a s}+1\right) L_{m}, L_{22}=L_{s}+L_{m} \\
& L_{25}=L_{52}=L_{m}, L_{44}=L_{55}=L_{r}+L_{m}
\end{aligned}
$$

and

$\mathbf{R}_{\mathbf{f}}^{*}=\left[\begin{array}{cccc}r^{*} & 0 & 0 & 0 \\ 0 & r_{s} & 0 & 0 \\ 0 & -\omega_{r} / \omega_{s} L_{m}^{*} & r r & -\omega_{s} / \omega_{s} L_{r} \\ \omega_{r} / \omega_{s} L_{m}^{*} & 0 & \omega_{r} / \omega_{s} L_{r} & r r\end{array}\right]$ with:

$$
\begin{aligned}
r^{*} & =r_{s} \cdot r_{s 11} \\
L_{m}^{*} & =L_{m} \cdot L_{14} \\
r_{s 11} & =\frac{1}{3}\left(2 g_{a s}+1\right)
\end{aligned}
$$

$g_{s a}$ is the percentage of the remaining un-shorted stator windings in stator phase $a$ (Chen and Zivanovic, 2009). These models will be utilized in the following simulation studies for simulating the healthy and the faulty induction motor operations.

\section{SET MEMBERSHIP IDENTIFICATION}

The method of Weighted Recursive Least Squares Set Membership Identification has been utilized efficiently in the fault detection and diagnosis in both cases of broken rotor bar and short circuit in stator winding for the three phase induction motor (Mustafa et al., 2012b). The objective of the Set Membership Identification technique (SMI) is the determination of a feasible recursively identified uncertainty parameter set that contains the nominal parameter vector and is consistent with a linearly parameterizable model, the measurement data and the a priori known bounded noise-error. In the SMI approach, instead of identifying directly the unknown parameter, the corresponding uncertainty bounds that include the nominal value are being calculated instead, while in each iteration the center of the uncertainty interval is equal with the current value of the identified parameter.
The $q-d$ model of the induction motor is being transformed into an ARMA system, which can be described in a generic form as:

$$
i_{j}(t)=\Phi_{j}(t)^{T} \hat{\theta}_{j}(t)+e_{j}(t)
$$

where $\hat{\theta}_{j}(t)$ is the identified parameter vector set and the subindex $j$ represents the current set that can be selected as one from: $[q s, d s]$. Moreover $\theta_{j}(t)$ contains the corresponding coefficients of the selected ARMA model and can be defined in the general case as:

$$
\hat{\theta}_{j}(t)=\left[F_{j, 1}(t), \ldots, F_{n, 1}(t), T_{j, 1}(t), \ldots, T_{m, 1}(t)\right]
$$

the regression vector $\Phi_{j}(t)$ is formulated as:

$$
\begin{aligned}
\Phi_{j}(t)= & {\left[-y_{j}(t-1), \ldots,-y_{j}(t-n), \ldots,\right.} \\
& \left.u_{j}(t+m-n-1), \ldots, u_{j}(t-n)\right]
\end{aligned}
$$

and the adopted ARMA model representation of the transfer function of induction motor will be denoted as:

$$
\frac{i_{j}}{V_{j}}=\frac{T_{j, 1} z^{m-1}+T_{j, 2} z^{m-2}+\ldots \ldots+T_{j, m}}{z^{n}+F_{j, 1} z^{n-1}+\ldots \ldots+F_{j, n}}
$$

The identified parameters presented in equation (3), are directly related with the motor parameters (resistance and inductance of the stator and rotor), while more details about the mathematical relationship between these parameters of induction motor in the healthy case could be found in (Mustafa et al., 2012b; Mustafa et al., 2012a), where $n, m \in Z^{+}$are the orders of the numerator and denominator for each considered transfer function and $\mu=n+m$.

In equation (2) the additive measurement noise is assumed to be bounded by $\gamma_{j} \in \mathfrak{R}^{+}$as:

$$
\gamma_{j}\left\|e_{j}(t)\right\|^{2} \leq 1, \forall t
$$

The core of the SMI technique is based on the Weighted Recursive Least Squares (WRLS) with a forgetting factor $(\lambda)$ for identifying the $\hat{\theta}_{j}$ motor's parameters and can be formulated by the following double recursions (Guastafsson, 2001) in the sample instance $t$ and for the MIMO case $j$ as:

$$
\begin{aligned}
\hat{\theta_{j}}(t)= & \hat{\theta_{j}}(t-1)+K_{j}(t)\left(y_{j}(t)-\Phi_{j}^{T}(t) \theta_{j}(t-1)\right) \\
K_{j}(t)= & P_{j}(t) \Phi_{j}(t)=P_{j}(t-1) \Phi_{j}(t)(\lambda \\
& \left.+\Phi_{j}^{T}(t) P_{j}(t-1) \phi_{j}(t)\right)^{-1} \\
P_{j}(t)= & \left(I-K_{j}(t) \Phi_{j}^{T}(t)\right) P_{j}(t-1) / \lambda \\
e_{j}(t)= & y_{j}(t)-\Phi_{j}^{T}(t) \theta_{j}(t-1) \\
G_{j}(t)= & \Phi_{j}^{T}(t) P_{j}(t-1) \Phi(t)
\end{aligned}
$$

In the SMI approach the uncertainty description is evolving with the time, as the better the knowledge of the parameters is, the smaller these bounds are, while 
as a fundamental property in SMI (Deller, 1989a), these bounds cannot be less than the assumed or computed range of noise corrupting the measurements. For calculating the upper and lower boundary of the identified parameters, the uncertainty bounds $\sigma_{j}(t)$, should be computed in every iteration and will become as:

$$
\sigma_{j}(t)=\sqrt{\operatorname{diag}\left(P_{j}(t)\right)}
$$

with the covariance matrix denoted as $C_{j}(t)=P_{j}(t)^{-1}$ and $P$ positive definite (Deller, 1989b; Le et al., 2008).

\section{FAULT DETECTION CONDITIONING}

Based on the SMI scheme, presented in Section 3 and on the uncertainty bounds in Eq. (4), for every identified parameter a hyper ellipsoid can be defined having as center the current values of the identified parameters from Eq. (2). In the following analysis, the notation for these identified values will be simplified to (Kurzhanskiy and Varaiya, 2008):

$$
\hat{\theta}_{j}(t)=\left[q_{1}(t) \ldots q_{n+m}(t)\right]
$$

and the ellipsoid will be considered as the set $\varepsilon(q, \sigma)$ in $R^{n+m}$ with center $q$ and shape matrix $\sigma$ as:

$$
\varepsilon(q, \sigma)=\left\{x \in R^{n} \mid\left\langle(x-q), \sigma^{-1}(x-q)\right\rangle \leq 1\right\}
$$

where $\sigma$ is positive definite, $\langle x, \sigma x\rangle>0$ for all nonzero $x \in R^{n+m}$ and with $\langle\cdot, \cdot\rangle$ to denote the inner product. The support function of a set $X \subseteq R^{n+m}$ and the support function of the ellipsoid in Eq. (6) will become as:

$$
\begin{aligned}
\rho(l \mid X) & =\sup _{x \in X}\langle x, l\rangle \\
\rho(l \mid \varepsilon(q, \sigma)) & =\langle l, q\rangle+(\langle l, \sigma l\rangle)^{\frac{1}{2}}
\end{aligned}
$$

Therefore, it is useful to give an alternative definition of an ellipsoid using the expression Eq. (8) as:

$$
\begin{aligned}
\varepsilon(q, \sigma)= & \left\{x \in R^{n} \mid\langle l, x\rangle \leq\langle l, q\rangle+\right. \\
& \left.\langle l, \sigma l\rangle^{\frac{1}{2}}\right\} \text { for all } l \in R^{n+m}
\end{aligned}
$$

where $\sigma$ is positive semidefinite, $\langle x, \sigma x\rangle \geq 0$ for all nonzero $x \in R^{n+m}$ (Kurzhanskiy and Varaiya, 2008). Given two hyper-ellipsoids $\varepsilon\left(q_{1}, \sigma_{1}\right)$ and $\varepsilon\left(q_{2}, \sigma_{2}\right)$, the distance between them is:

$$
\begin{aligned}
\operatorname{dist}\left(\varepsilon\left(q_{1}, \sigma_{1}\right), \varepsilon\left(q_{1}, \sigma_{1}\right)\right)= & \max _{\langle l, l\rangle=1}\left(-\rho\left(-l \mid \varepsilon\left(q_{1}, \sigma_{1}\right)\right)\right. \\
& -\left(\rho\left(l \mid \varepsilon\left(q_{2}, \sigma_{2}\right)\right)\right. \\
= & \max _{\langle l, l\rangle=1}\left(\left\langle l, q_{1}\right\rangle-\left(\left\langle l, \sigma_{1} l\right\rangle\right)^{\frac{1}{2}}\right. \\
& \left.-\left\langle l, q_{2}\right\rangle-\left(\left\langle l, \sigma_{2} l\right\rangle\right)^{\frac{1}{2}}\right)
\end{aligned}
$$

The intersection between two ellipsoids can be also transformed to a distance problem, which can be cast as Quadratically Constrained Quadratic Programming (QCQP) problem as: (Kurzhanskiy and Varaiya, 2008):

$$
\begin{array}{r}
\operatorname{dist}\left(\varepsilon\left(q^{o}, \sigma^{o}\right), \varepsilon\left(q^{f}, \sigma^{f}\right)\right)=\min \left\langle\left(x^{o}-y^{f}\right),\left(x^{o}-y^{f}\right)\right\rangle \\
\text { subject to } \\
\left\langle\left(q^{o}-x^{o}\right), \sigma^{o^{-1}}\left(q^{o}-x^{o}\right)\right\rangle \leq 1 \\
\left\langle\left(q^{f}-y^{f}\right), \sigma^{f^{-1}}\left(q^{f}-y^{f}\right)\right\rangle \leq 1
\end{array}
$$

with $x^{o}, x^{f}, y^{o}, y^{f} \in \mathfrak{R}^{n}$ points on the hyper-ellipsoids surfaces for the healthy and the faulty case respectively, with the following conditions:

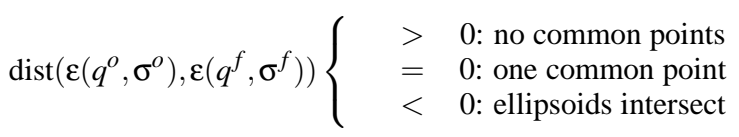

The proposed fault detection scheme is based only on geometrical properties of the structured parameters' uncertainty and more specifically is based on the online and fast computation of the recursively computed centers of ellipsoids and the corresponding distances between the centers. In all the performed geometrical calculations two ellipsoids are being considered: a) the nominal and converged ellipsoid of the error free identified motor model, denoted as $\varepsilon^{o}$, and b) the iteratively computed uncertainty ellipsoid $\varepsilon$ after parameters' convergence and the definition of the previous nominal ellipsoid $\varepsilon^{o}$. In Figure 1, two illustrative instances representing bounding ellipsoid uncertainty from the case of the healthy and faulty motor's operation are presented in a projection into a 2-dimensional frame, to allow a straight forward and comprehensive geometrical representation, without any loss of generality.

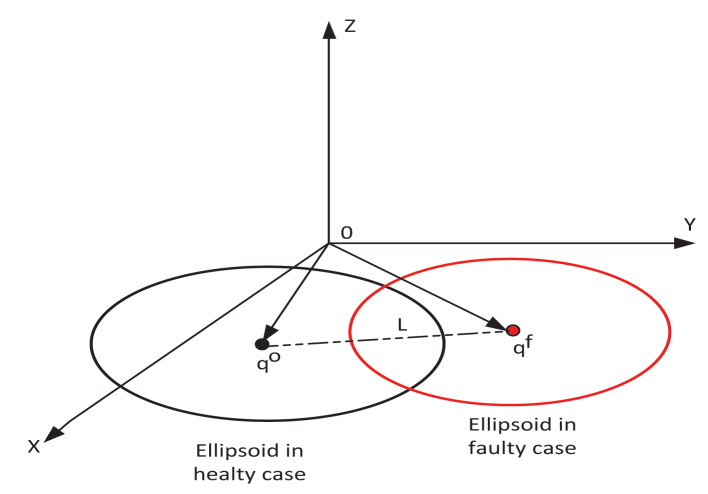

Figure 1: Projection of ellipsoid intersection in X-Y plane.

In this Figure, the general case of intersection and shifting of centers between the two ellipsoids has been 
presented also. while the distance $L(t) \in \mathfrak{R}^{n+m}$ between the centers has been denoted as:

$$
L(t)=\left[\left(q_{1}^{o}-q_{1}^{f}\right)^{2}+\left(q_{2}^{o}-q_{2}^{f}\right)^{2}+\ldots .+\left(q_{n+m}^{o}-q_{n+m}^{f}\right)^{2}\right]^{\frac{1}{2}}
$$

In the case of a stator winding short circuit, the values of the identified parameters and the corresponding uncertainty bounds will drift from their converged values, which has a direct effect and change on the center and the direction of the bounding uncertainty $\mu$-dimensional hyper ellipsoids at each sample time (iteration) and thus a fault detection scheme can be directly established based on a straight forward inspection of geometrical properties for the bounding ellipsoids as it is being presented in Figure 2.

The initialization phase of the proposed fault detection algorithm assumes that the SMI scheme has converged in identifying the nominal parameters of the adopted model for the induction motor and thus the corresponding bounds on the parametric uncertainty intervals have been also converged. In the sequel the SMI scheme continuous the online identification of the parameters, while constructing the sequential bounding uncertainty $\mu-D$ ellipsoids. Upon a short circuit fault the upcoming online calculated $\mu-\mathrm{D}$ bounding ellipsoids will present a change in their volume and an intersection will occur with the nominal defined converged $\mu-\mathrm{D}$ ellipsoid volume, which is a direct indication of a fault. In case that there is no intersection the fault detection algorithm will continue to operate, while checking the existence of such an intersection. The parametric drift after the event of the fault will also produce a corresponding drift on the ellipsoid's center and thus the distance among ellipsoid centers are being also tracked by calculating the value of $L$. In case that this distance is not close to 0 , then in combination with the ellipsoid intersection, these two conditions will lead to a fault detection instance.

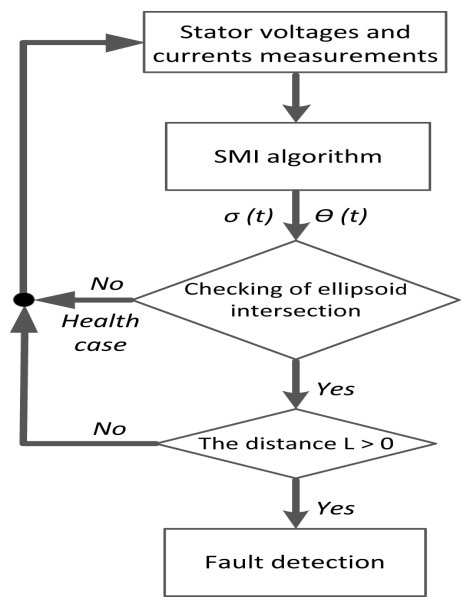

Figure 2: Flowchart of fault detection conditions.

\section{SIMULATION RESULTS}

The suggested scheme for fault detection is being evaluated on a model of three phase induction motor having the parameters depicted in Table 1. The presented results examine the application of the proposed geometrical analysis on two cases of short circuit stator winding with $2 \%$, and $5 \%$ faults.

Table 1: Induction Motor Parameters.

\begin{tabular}{|l|c||c|c|}
\hline Pole Numbers & 4 & $r_{s}$ & 0.0616 per unit \\
\hline Input Voltage & $240 \mathrm{~V}$ & $r_{r}$ & 0.0753 per unit \\
\hline Frequency & $50 \mathrm{~Hz}$ & $\mathrm{~J}$ & $0.00155 \mathrm{Kg} . \mathrm{m}$ \\
\hline$L_{r}$ & 0.019 per unit & $L_{s}$ & 0.019 per unit \\
\hline$L_{m}$ & 0.01833 per unit & & \\
\hline
\end{tabular}

The identified parameters and uncertainty bounds in the healthy and faulty case of $2 \%$ short circuit are presented in figures 3 and 4, where the fault occurred at the $10^{5}$ sample time instance and the change in the motor parameters are obvious because of this stator fault. In Figure 5 the iterative evolution of
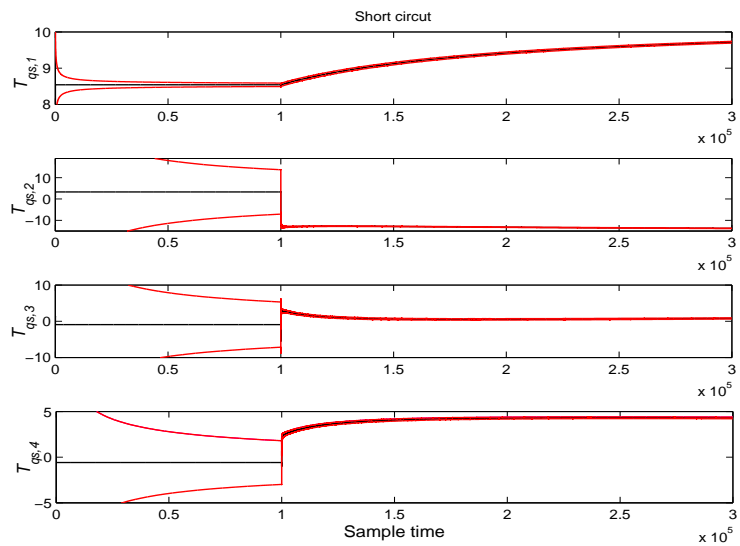

Figure 3: SMI based identified parameters for $T_{q s, i}$ and corresponding uncertainty bounds.

the ellipsoid volume (bounding uncertainty), during identification in the healthy case and for a projection based on the selected triplet (center of ellipsoid) of $T_{q s, 1}, T_{q s, 2}, T_{q s, 3}$ is being presented at different time indexes. From the obtained results it can be observed that the ellipsoids volume is monotonically decreased without any intersections taking place at the different time instances. This result and the presented bounding uncertainty are in fully accordance with the results presented in Figure 5.

In Figures 6 and 7 the uncertainty ellipsoids in the nominal case and after the occurrence of the fault at different sample times for the same case of considering the triplet of $T_{q s, 1}, T_{q s, 2}, T_{q s, 3}$ identified parameters, for both the cases of $2 \%$ and $5 \%$ of stator wind- 

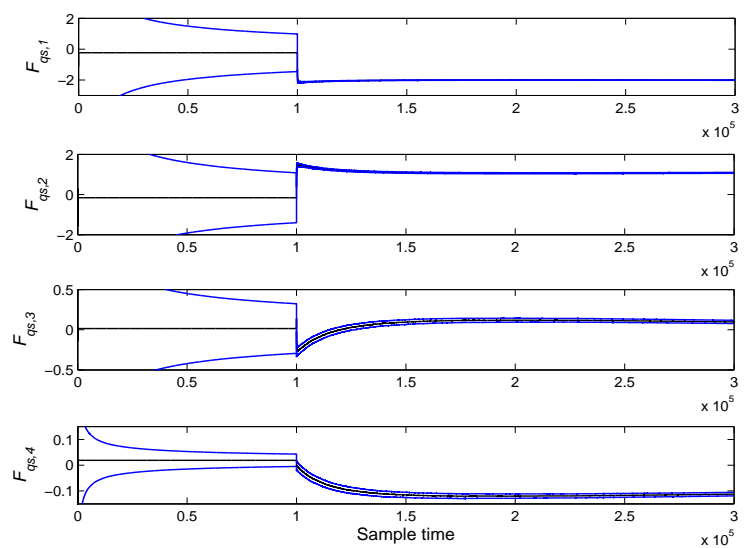

Figure 4: SMI based identified parameters for $F_{q s, i}$ and corresponding uncertainty bounds.

ing short circuit correspondingly are being presented.

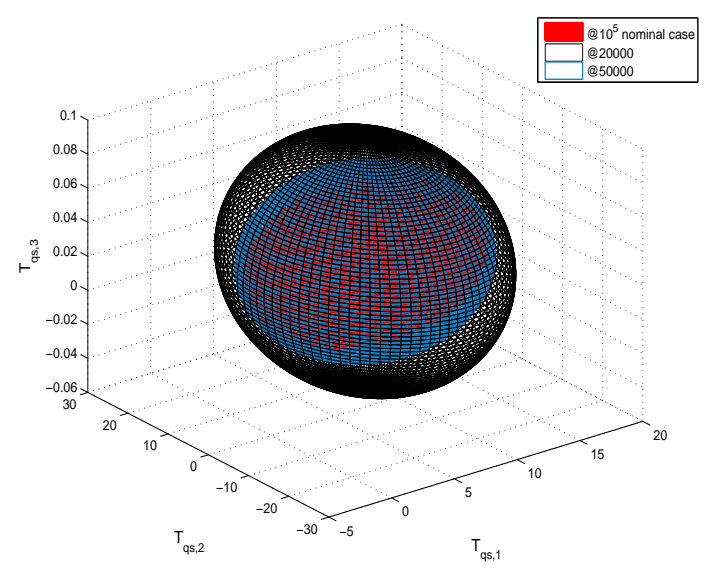

Figure 5: Ellipsoid in healthy case at different sample time.

The centers of ellipsoids and the corresponding uncertainty bounds will drift from their converged healthy value at each sample time. The ellipsoids intersection between the nominal case and the faulty case has been easily tracked in the proposed geometrical approach to fault detection, while this drift will evolve after fault's occurrence. In Figure 8 it is also presented the geometrical drift in the bounding ellipsoids and the corresponding intersection, for the case where a different triplet have been considered of a center $T_{q s, 1}, F_{q s, 2}, T_{q s, 4}$ for the case of $2 \%$ short circuit.

Finally the distance $L$ in the case that the whole $(\mu)$-dimensional hyper ellipsoid is being considered during the healthy operation and after the fault occurrence, for the cases of $2 \%$, and $5 \%$ short circuit are being presented in Figure 10. As it can be presented, the hyper distance will be increased after the fault occurrence and it will become greater than zero as shown in figures (9).

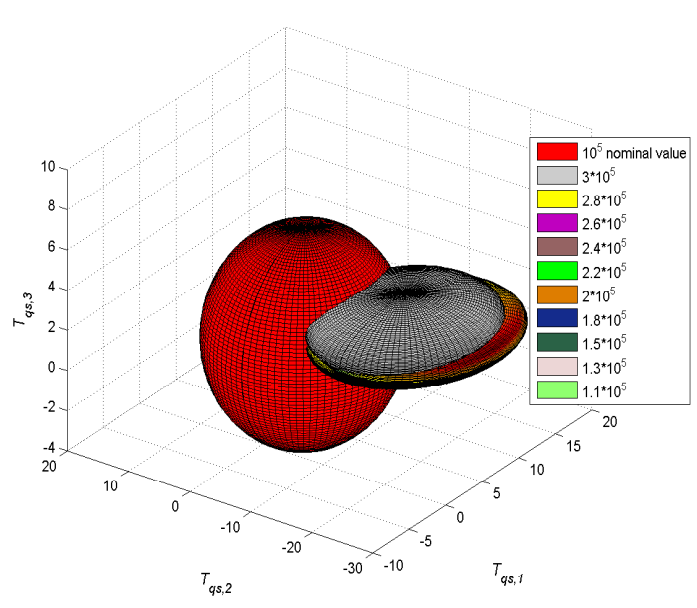

Figure 6: Ellipsoid interaction between healthy and faulty case (2\% short circuit).

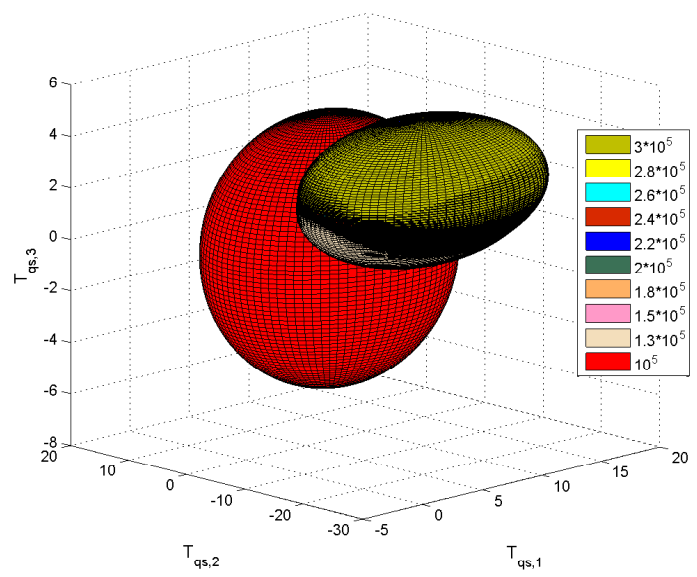

Figure 7: Ellipsoid interaction between healthy and faulty case (5\% short circuit).

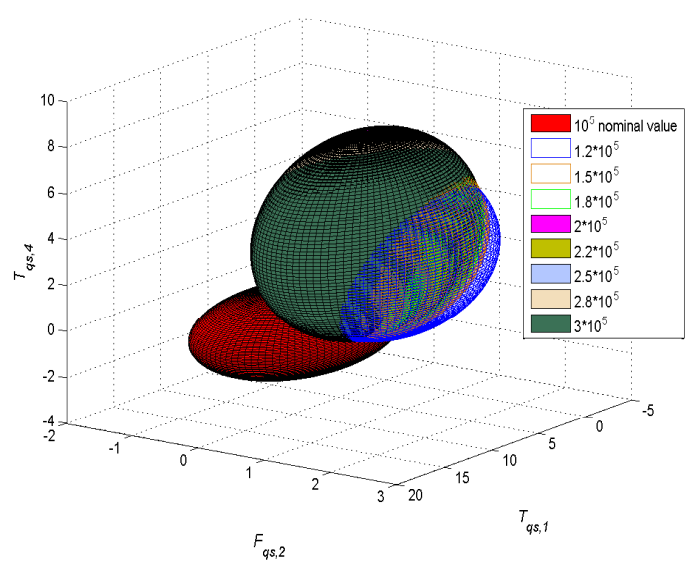

Figure 8: Ellipsoid interaction between healthy and faulty case (2\% short circuit). 


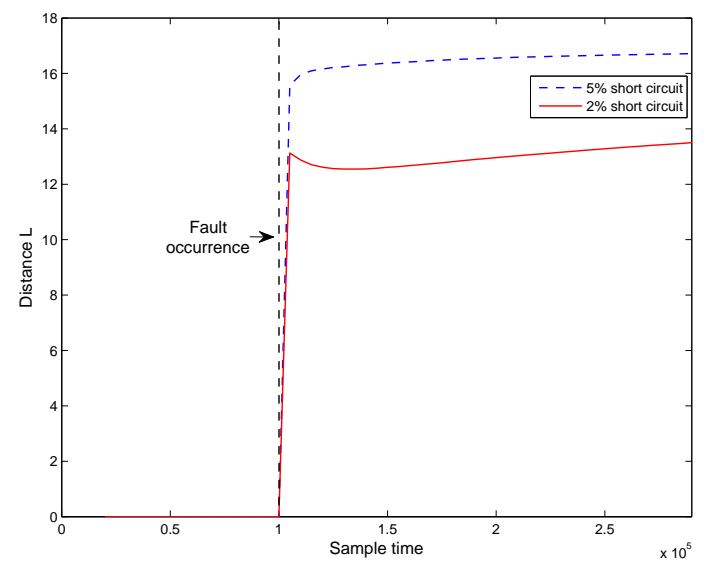

Figure 9: The distance $L$ in healthy and faulty case (2\%, $5 \%$ short circuit).

\section{CONCLUSIONS}

In this article a fault detection scheme for different percentage of stator winding short circuit in one phase of three phase induction motors is presented. The motor's model was identified by the utilization of a Least Squares Set Membership Identification (SMI) algorithm, where additional to the identified parameters, confidence intervals have been calculated, These intervals in an $\mu$-dimensional space can be represented as hyper-ellipsoids having as a center the identified parameters' vector and thus a geometrical fault detection scheme has been proposed, which relied on the calculation of the distance among centers of hyperellipsoids and the corresponding intersections. Extended simulation results were presented that proven the efficiency of the suggested scheme.

\section{REFERENCES}

A. Widodo, a. B. Y. (2008). Wavelet support vector machine for induction machine fault diagnosis based on transient current signal. Expert Systems with Applications, 35:307316.

Aydin, I., Karakose, M., and Aki, E. (2011). A new method for early fault detection and diagnosis of broken rotor bars. Energy Conversion and Management, 52(4):1790-1799.

B. Mirafzal, N. A. O. D. (2006). On innovative methods of induction motor interturn and broken-bar fault diagnostics. IEEE TRANSACTIONS ON INDUSTRY APPLICATIONS, 42(2).

Bachi, S., Tnani, S., Trigeassou, J., and Champenois, G. (2006). Diagnosis by parameter estimation of stator and rotor faults occurring in induction ma- chines. IEEE Transactions on Industrial Electronics, 53(3):963-973.

Chen, S. and Zivanovic, R. (2009). Modelling and simulation of stator and rotor fault conditions in induction machines for testing fault diagnostic techniques. European Transactions On Electrical Power, 20:611629.

Deller, J. (1989a). Set membership identification in digital signal processing. IEEE ASSP Magazine, 6(4):4-20.

Deller, J. R. (1989b). Set membership identification in digital signal processing. IEEE ASSP Magazine, 6(4):420.

F. Jawad, O. M. (2009). Different indexes for eccentricity faults diagnosis in three-phase squirrel-cage induction motors a review. Mechanical Sustem and Signal Processing, 19:213.

Gaeid, K. S. and Mohamed, H. A. F. (2010). Bibliography on induction motors faults detection and diagnosis. Australian Journal of Basic and Applied Sciences, 4(2):227-246.

Guastafsson, F. (Sep.2001). Adaptive Filtering and Change Detection.

Kurzhanskiy, A. A. and Varaiya, P. (2008). Ellipsoidal toolbox manual.

Le, K., Huang, Z., Moon, C., and Tzes, A. (2008). Fault detection based on orthotopic set membership identification for robot manipulators. Proceedings of the 17th World Congress The International Federation of Automatic Control, Seoul, Korea,2008, pages 73447349.

Mustafa, M. O., Nikolakopoulos, G., and Guastafsson, T. (2012a). Stator winding short circuit fault detection based on set membership identification for three phase induction motors. Proceedings of IEEE, 20th Mediterranean Conference on Control and Automation, MED12, pages 290-296.

Mustafa, M. O., Nikolakopoulos, G., and Gustafsson, T. (2012b). A fault diagnosis scheme for three phase induction motors based on uncertainty bounds. Proceedings Of The 38th Annual Conference of the IEEE Industrial Electronics Society IECON, pages 16061611.

Nandi, S. and Toliyat, H. (2005). Condition monitoring and fault diagnosis of electrical machines-a review. IEEE Transactions on Energy Conversion, 20(4):719-729.

Vas, P. (1992). Electrical Machines and Drives.

Widodo, A., Yang, B. S., and Han, T. (2007). Combination of independent component analysis and support vector machines for intelligent faults diagnosis of induction motors. Expert Systems with Applications, 32:299312. 